Article

\title{
Anxiety, Post-Traumatic Stress, and Burnout in Health Professionals during the COVID-19 Pandemic: Comparing Mental Health Professionals and Other Healthcare Workers
}

\author{
Isabella Giulia Franzoi 1,*(D), Antonella Granieri ${ }^{1}$, Maria Domenica Sauta ${ }^{1}$, Monica Agnesone ${ }^{2}$, Marco Gonella ${ }^{1,2}$, \\ Roberto Cavallo $^{3}$, Piergiorgio Lochner ${ }^{4}$, Nicola Luigi Bragazzi ${ }^{5}$ iD and Andrea Naldi ${ }^{3,6}$
}

check for updates

Citation: Franzoi, I.G.; Granieri, A.; Sauta, M.D.; Agnesone, M.; Gonella, M.; Cavallo, R.; Lochner, P.; Bragazzi, N.L.; Naldi, A. Anxiety, Post-Traumatic Stress, and Burnout in Health Professionals during the COVID-19 Pandemic: Comparing Mental Health Professionals and Other Healthcare Workers. Healthcare 2021, 9, 635. https://doi.org/ $10.3390 /$ healthcare 9060635

Academic Editors: Fabrizia Giannotta and Yunhwan Kim

Received: 7 May 2021

Accepted: 25 May 2021

Published: 27 May 2021

Publisher's Note: MDPI stays neutral with regard to jurisdictional claims in published maps and institutional affiliations.

Copyright: (c) 2021 by the authors. Licensee MDPI, Basel, Switzerland. This article is an open access article distributed under the terms and conditions of the Creative Commons Attribution (CC BY) license (https:/ / creativecommons.org/licenses/by/ $4.0 /)$.
1 Department of Psychology, University of Turin, 10124 Turin, Italy; antonella.granieri@unito.it (A.G.); mariadomenica.sauta@unito.it (M.D.S.); marco.gonella@unito.it (M.G.)

2 SS Psychology, Local Health Authority "Città di Torino", 10143 Turin, Italy; monica.agnesone@aslcittaditorino.it

3 Neurology Unit, San Giovanni Bosco Hospital, 10154 Turin, Italy; roberto.cavallo@aslcittaditorino.it (R.C.); naldi.andrea@yahoo.it (A.N.)

4 Department of Neurology, Saarland University Medical Center, 66421 Homburg, Germany; piergiorgio.lochner@gmail.com

5 Laboratory for Industrial and Applied Mathematics (LIAM), Department of Mathematics and Statistics, York University, Toronto, ON M3J 1P3, Canada; robertobragazzi@gmail.com

6 Department of Neuroscience "Rita Levi Montalcini", University of Turin, 10126 Turin, Italy

* Correspondence: isabellagiulia.franzoi@unito.it; Tel.: +39-0116703062

Abstract: The psychological impact of the pandemic on healthcare workers has been assessed worldwide, but there are limited data on how mental health professionals (MHPs) have been affected. Thus, this paper aims to investigate anxiety, post-traumatic stress, and burnout in a sample of MHPs. We conducted a descriptive, cross-sectional study on 167 participants: 56 MHPs, 57 physicians working closely with COVID-19 patients, and 54 physicians not working closely with such patients. MHPs reported good overall mental health. Most MHPs reported no post-traumatic stress, and their scores were significantly lower compared to HPs working closely with COVID-19 patients. MHPs' hyperarousal scores were also significantly lower compared to HPs working closely with COVID-19 patients, while their intrusion scores were statistically significantly lower than those of all other professionals. Multivariable logistic regressions showed that MHPs had lower odds of exhibiting state anxiety and low personal accomplishment compared to HPs not working closely with COVID-19 patients. In sum, MHPs seem to show almost preserved mental health. Thus, given the high mental healthcare demand during a pandemic, it would be useful to rely on these professionals, especially for structuring interventions to improve and support the mental health of the general population and other healthcare workers.

Keywords: COVID-19; mental health; mental health professionals; pandemic; distress; risk-factors; protective factors

\section{Introduction}

Since the novel coronavirus disease (COVID-19) emerged in China at the end of 2019 and was subsequently classified as a pandemic by the World Health Organization on 11 March 2020, its impact on society has been enormous. Worldwide, authorities have had to take extreme actions to contain its spread. In the initial absence of a vaccine, quarantine has been implemented as one of the most effective measures to reduce transmission risk. The disease itself, fear of contagion, and the different containment measures have significantly affected people's occupational and social lives, as well as their physical health and psychological wellbeing [1]. A pandemic can place severe strain on general mental health resources, potentially leading to untreated mental health issues [2-4]. A large 
range of psychological outcomes emerged during the virus outbreak and continue to be experienced: stress, anxiety, depression, and fear of getting sick or dying [5,6]. Moreover, symptoms of post-traumatic stress disorder or complicated grief disorder have emerged, as they often do in the aftermath of global emergencies or disasters [7-11]. Prolonged social distancing, social isolation, and the illness or loss of family members can substantially exacerbate mental health issues [5,12-16]. In particular, recent studies show that people held in isolation or quarantine experienced significant levels of anxiety, anger, confusion, and stress $[17,18]$. Moreover, fear of infection can cause pervasive and persistent worries over one's health and the potential risk of infecting others, especially loved ones [19,20].

Although a pandemic affects the entire population, individuals that are most exposed have a higher risk of developing mental health problems [21,22]. Research conducted during the outbreak of MERS and SARS showed that healthcare workers (HCWs) had the greatest risk of experiencing psychological distress [23]. The feeling of uncertainty, threat to health, and somatic symptoms were particularly prevalent [24]. The psychological impact of the COVID-19 pandemic on frontline HCWs has been assessed worldwide through numerous studies. During the SARS-CoV-2 pandemic, HCWs are facing aggravated psychological pressure and seem to be more severely affected by mental health issues [25-27] and indirect traumatization [21] than other occupational groups. In particular, they seem to experience depression, anxiety, traumatic stress, avoidance, and burnout [28]. Depression and anxiety symptoms reported by HCWs range from moderate to severe; moreover, the literature reveals that anxiety is positively correlated with the total amount of stress and workload [29,30]. Isolation from relatives, working in high-risk wards, fear, and feeling guilt over contributing to contagion have all been reported as major causes of trauma [31].

However, it seems that too little attention has been paid to the pandemic's impact on mental health professionals (MHPs); to date, only Joshi and Sharma [32] have preliminarily underlined those stressors that put MHPs at risk of burnout, such as staff shortage and workload increase. Therefore, how the pandemic has affected the psychological wellbeing of MHPs remains largely unknown.

Thus, this paper investigates anxiety, post-traumatic distress, and burnout in a sample of MHPs, comparing their distress to that experienced by other health professionals (HPs), including those working closely and those not working closely with COVID-19 patients. We aim to detect potential sociodemographic, family, and occupational factors influencing the emotional impact of the pandemic.

\section{Materials and Methods}

\subsection{Study Design and Participants}

We conducted a descriptive, cross-sectional study through a web-based survey. Enrollment progressively included HCWs of four hospitals in Turin, Piedmont, North Italy, all directly engaged in managing COVID-19 patients.

The survey was sent by email to all HCWs of the four hospitals on 27 April 2020, explaining that study recruitment would end at midnight of the day on which the target sample size was achieved. The minimum sample size $(n=377)$ was calculated through the formula $N=Z^{2} \times p(1-p) / \alpha^{2}$ (considering a margin of error of $5 \%(\alpha=0.05)$, a $95 \%$ confidence interval $(Z=1.96)$, and a population proportion of $50 \%(p=0.50))$. However, to ensure meaningful subgroup analysis, the target sample size was doubled. Hence, at midnight of 1 May 2020, the survey was closed after a total of 962 HCWs had completed the questionnaire. This study focused on the experience of MHPs (including psychologists and psychiatrists, $n=56$ ), compared with that of physicians who worked closely with COVID-19 patients receiving high-intensity care $(n=57)$ and physicians who reported none or only occasional contact with COVID-19 patients receiving low-intensity care $(n=54)$. Details of the different specializations of participating physicians are provided in Table 1. Psychological data regarding the entire population of physicians and nurses have previously been published [30]. 
Table 1. Study participants.

\begin{tabular}{|c|c|c|}
\hline & $\mathbf{n}$ & $\%$ \\
\hline \multicolumn{3}{|l|}{ Mental health professionals } \\
\hline Psychiatrists & 16 & 28.57 \\
\hline Psychologists & 40 & 71.43 \\
\hline Total & 56 & 100.00 \\
\hline \multicolumn{3}{|c|}{ Physicians working closely with COVID-19 patients } \\
\hline Anesthesiologists & 28 & 49.12 \\
\hline Infectious diseases specialists & 15 & 26.32 \\
\hline ED physicians & 14 & 24.56 \\
\hline Total & 57 & 100.00 \\
\hline \multicolumn{3}{|c|}{ Physicians not working closely with COVID-19 patients } \\
\hline Pathologists & 2 & 3.70 \\
\hline General Surgeons & 3 & 5.56 \\
\hline Endocrinologists & 7 & 12.96 \\
\hline Gynecologists & 4 & 7.41 \\
\hline Preventive medicine specialists & 8 & 14.81 \\
\hline Forensic pathology physicians & 4 & 7.41 \\
\hline Ophthalmologists & 2 & 3.70 \\
\hline Dentists & 3 & 5.56 \\
\hline Oncologists & 2 & 3.70 \\
\hline Radiologists & 4 & 7.41 \\
\hline Urologists & 2 & 3.70 \\
\hline Other physicians * & 13 & 24.07 \\
\hline Total & 54 & 100.00 \\
\hline
\end{tabular}

*E.g., occupational health physicians, rehabilitation physicians, neurosurgeons, orthopedics, clinical pathologists, nutritionists.

Recruitment was undertaken in an especially demanding period of the COVID-19 health crisis in Italy: from March 10 to 3 May 2020, a nationwide quarantine was imposed, and the total numbers of COVID-19-confirmed cases exceeded 26,000 in Piedmont and 207,000 in Italy [33].

\subsection{Survey Instrument}

A web-based survey was developed to investigate burnout, anxiety, and post-traumatic stress in a sample of HCWs during the COVID-19-related health emergency in Italy, and to detect potential sociodemographic, family, and occupational factors. We chose this data collection method to ensure social distancing, as required by the Italian government at the time of the enrollment. The questionnaire was completed anonymously and administrated in Italian. The survey was conducted through Google Forms, a survey-administration app in the Google Drive office suite.

The first page of the web survey provided study details and required all participants to give informed consent by ticking the box marked "agree" before being allowed to access the next page. The survey then investigated sociodemographic, family, working, and psychological characteristics of the recruited sample. Sociodemographic data included age range, sex, and working position. Family data included having children, living situation before the pandemic (with or without partner), and occurrence of family division due to COVID-19 for safety reasons and/or fear of contagion. Working data included contact 
frequency with COVID-19 patients (frequent, rare, or none), the extent of work changes related to the pandemic, and changes in the relationship with patients (improved, worsened, or unchanged). Finally, psychological data were investigated through a pool of self-report measures assessing anxiety, post-traumatic stress, and occupational burnout, previously validated for the Italian population.

\subsection{Outcome Measures}

The self-report psychological measures comprised the State-Trait Anxiety InventoryY (STAI-Y) [34,35], the Impact of Event Scale-Revised (IES-R) [36,37], and the Maslach Burnout Inventory (MBI) [38,39].

The STAI-Y is a self-report inventory aimed at assessing both state (STAI-Y1) and trait (STAI-Y2) anxiety. State anxiety is the feeling of insecurity and helplessness in the face of a perceived threat that may lead to concern or avoidance, while trait anxiety is the tendency to experience and report, across many situations, negative emotions such as fears, worries, and anxiety. Each dimension comprises 20 non-overlapping questions. Participants are asked to rate how accurately each item describes them on a four-point scale $(1=$ "almost never" to 4 = "almost always"). Total scores for both scales range from 20 to 80 . Consistent with previous research $[40,41]$, we used a cut-off of 40 to evaluate the presence or absence of state and trait anxiety.

The IES-R is widely used to measure distress symptoms connected to traumatic events. It comprises 22 items assessing symptoms of avoidance (i.e., keeping away from particular activities, thoughts, etc. because of their anticipated negative consequences), intrusion (i.e., inability to keep memories of the event from returning), and hyperarousal (i.e., tendency to be easily startled, constant feeling that danger or disaster is nearby, inability to concentrate, irritability, or even violent behavior). Participants are asked to rate how distressing each item has been during the past week on a five-point Likert scale $(0=$ "not at all" to $4=$ "extremely"). The total score ranges from 0 to 88 , with a score of 33 or higher indicating possible presence of post-traumatic symptoms [42]. In particular, 0-23 indicates the absence of post-traumatic symptomatology, 24-32 indicates clinically detectable post-traumatic symptomatology, 33-36 indicates the possible presence of posttraumatic symptoms, and $\geq 37$ indicates severe post-traumatic symptomatology.

The MBI is a measure of occupational burnout comprising three scales: emotional exhaustion (EE, nine items), examining the feeling of being emotionally bitter and exhausted from work; depersonalization (DP, five items), which measures a cold and impersonal response; personal accomplishment (PA, eight items), assessing the feeling of own competence and desire to succeed in working with others. High EE and DP scores indicate a higher level of burnout, while high PA scores indicate a lower level of burnout. Participants are asked to rate how often they experience what each item describes on a seven-point Likert scale $(0=$ "never" to $6=$ "daily"). Consistent with previous research [43-45], we considered the level of burnout to be high with $\mathrm{EE} \geq 24, \mathrm{PA} \leq 29$, and DP $\geq 9$, moderate with $\mathrm{EE}=15-23, \mathrm{PA}=30-36$, and $\mathrm{DP}=4-8$, and low with $\mathrm{EE} \leq 14, \mathrm{PA} \geq 37$, and $\mathrm{DP} \leq 3$.

\subsection{Statistical Analysis}

Data analyses were conducted using the Statistical Package for the Social Sciences (SPSS; IBM Corp., Armonk, NY, USA) version 26. We first computed skewness and kurtosis, and tested for normality with a Kolmogorov-Smirnov test. Since no continuous variables were normally distributed, they were all expressed as median and interquartile range (IQR) values, while categorical variables were expressed as frequency and percentage values. For continuous variables, differences between independent groups were evaluated using a Kruskal-Wallis H test. Subsequently, pairwise comparisons were performed using Dunn's [46] procedure with Bonferroni correction for multiple comparisons. Categorical variables were compared using a chi-square test. Post-hoc analyses with Bonferroni corrections were conducted using adjusted residuals. Then, five multivariable logistic regressions were performed to ascertain the effects of possible risk factors on the likelihood of showing 
state anxiety, moderate-to-severe post-traumatic symptoms, high emotional exhaustion, high depersonalization, and low personal accomplishment. All tests were two-tailed, and we set the statistical significance threshold at $p \leq 0.05$.

\section{Results}

\subsection{Participants}

As reported in Table 1, the final sample comprised 167 participants. Among the $56 \mathrm{MHPs}, 40(71.43 \%)$ were psychologists and $16(28.57 \%)$ were psychiatrists. Among the 57 physicians working closely with COVID-19 patients, $28(49.12 \%)$ were anesthesiologists, $15(26.32 \%)$ were infectious diseases specialists, and 14 (24.56\%) were ER physicians. The 54 physicians not working closely with COVID-19 patients had various specializations: for example, eight (14.81\%) were preventive medicine specialists and seven $(12.96 \%)$ were endocrinologists.

As Table 2 shows, no MHPs reported frequent contact with COVID-19 patients.

Table 2. Contact with COVID-19 patients.

\begin{tabular}{|c|c|c|c|c|c|c|}
\hline & \multicolumn{2}{|c|}{$\begin{array}{c}\text { Mental Health } \\
\text { Professionals }(n=56)\end{array}$} & \multicolumn{2}{|c|}{$\begin{array}{l}\text { Physicians Working } \\
\text { Closely with COVID-19 } \\
\text { Patients }(n=57)\end{array}$} & \multicolumn{2}{|c|}{$\begin{array}{l}\text { Physicians not Working } \\
\text { Closely with COVID-19 } \\
\text { Patients }(n=54)\end{array}$} \\
\hline & $n$ & $\%$ & $n$ & $\%$ & $n$ & $\%$ \\
\hline \multicolumn{7}{|c|}{$\begin{array}{l}\text { Contact with COVID-19 patients } \\
\text { in the last two months }\end{array}$} \\
\hline None & 47 & 83.93 & 0 & 0.00 & 24 & 44.44 \\
\hline Rare & 9 & 16.07 & 0 & 0.00 & 30 & 55.56 \\
\hline Frequent & 0 & 0.00 & 57 & 100.00 & 0 & 0.00 \\
\hline
\end{tabular}

As reported in Table 3, most MHPs were female $(48,85.71 \%)$ and aged $\geq 41$ years (50, $89.28 \%$ ).

Table 3. Differences between categorical variables.

\begin{tabular}{|c|c|c|c|c|c|c|c|c|c|}
\hline & \multicolumn{2}{|c|}{$\begin{array}{l}\text { Mental Health } \\
\text { Professionals } \\
\quad(n=56)\end{array}$} & \multicolumn{2}{|c|}{$\begin{array}{c}\text { Physicians } \\
\text { Working Closely } \\
\text { with COVID-19 } \\
\text { Patients }(n=57)\end{array}$} & \multicolumn{2}{|c|}{$\begin{array}{c}\text { Physicians not } \\
\text { Working Closely } \\
\text { with COVID-19 } \\
\text { Patients }(n=54)\end{array}$} & \multirow[b]{2}{*}{$x^{2}$} & \multirow[b]{2}{*}{$d f$} & \multirow[b]{2}{*}{$p$} \\
\hline & $n$ & $\%$ & $n$ & $\%$ & $n$ & $\%$ & & & \\
\hline \multicolumn{10}{|c|}{ Socio-Demographic Characteristics } \\
\hline Age range & & & & & & & 43.52 & 4 & $<0.001$ \\
\hline$\leq 40$ years & 6 & 10.71 & 29 & 50.88 & 7 & 12.96 & & & \\
\hline $41-50$ years & 27 & 48.21 & 16 & 28.07 & 11 & 20.37 & & & \\
\hline$\geq 50$ years & 23 & 41.07 & 12 & 21.05 & 36 & 66.67 & & & \\
\hline Gender & & & & & & & 2.54 & 2 & 0.282 \\
\hline Female & 48 & 85.71 & 38 & 66.67 & 31 & 57.41 & & & \\
\hline Male & 8 & 14.29 & 19 & 33.33 & 23 & 42.59 & & & \\
\hline \multicolumn{10}{|c|}{ Family Conditions } \\
\hline Children & & & & & & & 2.54 & 2 & 0.282 \\
\hline No & 19 & 33.93 & 24 & 42.11 & 15 & 27.78 & & & \\
\hline Yes & 37 & 66.07 & 33 & 57.89 & 39 & 72.22 & & & \\
\hline
\end{tabular}


Table 3. Cont.

\begin{tabular}{|c|c|c|c|c|c|c|c|c|c|}
\hline & \multicolumn{2}{|c|}{$\begin{array}{l}\text { Mental Health } \\
\text { Professionals } \\
\quad(n=56)\end{array}$} & \multicolumn{2}{|c|}{$\begin{array}{c}\text { Physicians } \\
\text { Working Closely } \\
\text { with COVID-19 } \\
\text { Patients }(n=57)\end{array}$} & \multicolumn{2}{|c|}{$\begin{array}{c}\text { Physicians not } \\
\text { Working Closely } \\
\text { with COVID-19 } \\
\text { Patients }(n=54)\end{array}$} & \multirow[b]{2}{*}{$x^{2}$} & \multirow[b]{2}{*}{$d f$} & \multirow[b]{2}{*}{$p$} \\
\hline & $n$ & $\%$ & $n$ & $\%$ & $n$ & $\%$ & & & \\
\hline Living alone before the pandemic & & & & & & & 2.47 & 2 & 0.291 \\
\hline No & 49 & 87.50 & 46 & 80.70 & 49 & 90.74 & & & \\
\hline Yes & 7 & 12.50 & 11 & 19.30 & 5 & 9.26 & & & \\
\hline Family separation due to COVID-19 & & & & & & & 5.88 & 2 & 0.053 \\
\hline No & 40 & 81.63 & 29 & 63.04 & 40 & 81.63 & & & \\
\hline Yes & 9 & 18.37 & 17 & 36.96 & 9 & 18.37 & & & \\
\hline \multicolumn{10}{|l|}{ Working Conditions } \\
\hline Work changes due to COVID-19 & & & & & & & 0.47 & 6 & 0.998 \\
\hline Not at all & 14 & 25.00 & 13 & 22.81 & 15 & 27.78 & & & \\
\hline Slightly & 14 & 25.00 & 16 & 28.07 & 14 & 25.93 & & & \\
\hline Considerably & 20 & 35.71 & 20 & 35.09 & 18 & 33.33 & & & \\
\hline Extremely & 8 & 14.29 & 8 & 14.04 & 7 & 12.96 & & & \\
\hline $\begin{array}{l}\text { Changes in the relationship with } \\
\text { patients due to COVID-19 }\end{array}$ & & & & & & & 11.11 & 4 & 0.025 \\
\hline Not at all & 7 & 12.50 & 11 & 19.30 & 12 & 22.22 & & & \\
\hline Improved & 14 & 25.00 & 7 & 12.28 & 19 & 35.19 & & & \\
\hline Worsened & 35 & 62.50 & 39 & 68.42 & 23 & 42.59 & & & \\
\hline \multicolumn{10}{|l|}{ Psychological Distress } \\
\hline STAI-Y Trait Anxiety & & & & & & & 0.78 & 2 & 0.676 \\
\hline No trait anxiety & 19 & 33.93 & 15 & 26.32 & 16 & 29.63 & & & \\
\hline Trait anxiety & 37 & 66.07 & 42 & 73.68 & 38 & 70.37 & & & \\
\hline STAI-Y State Anxiety & & & & & & & 5.63 & 2 & 0.060 \\
\hline No state anxiety & 38 & 67.86 & 27 & 47.37 & 27 & 50.00 & & & \\
\hline State anxiety & 18 & 32.14 & 30 & 52.63 & 27 & 50.00 & & & \\
\hline IES-R TOT & & & & & & & 11.68 & 6 & 0.069 \\
\hline Normal & 32 & 57.14 & 24 & 42.11 & 31 & 57.41 & & & \\
\hline Mild & 13 & 23.21 & 8 & 14.04 & 5 & 9.26 & & & \\
\hline Moderate & 2 & 3.57 & 5 & 8.77 & 6 & 11.11 & & & \\
\hline Severe & 9 & 16.07 & 20 & 35.09 & 12 & 22.22 & & & \\
\hline MBI Emotional Exhaustion & & & & & & & 4.87 & 4 & 0.301 \\
\hline Low & 29 & 51.79 & 20 & 35.09 & 20 & 37.04 & & & \\
\hline Medium & 11 & 19.64 & 16 & 28.07 & 11 & 20.37 & & & \\
\hline High & 16 & 28.57 & 21 & 36.84 & 23 & 42.59 & & & \\
\hline MBI Depersonalization & & & & & & & 23.54 & 4 & $<0.001$ \\
\hline Low & 40 & 71.43 & 16 & 28.07 & 27 & 50.00 & & & \\
\hline Medium & 11 & 19.64 & 21 & 36.84 & 11 & 20.37 & & & \\
\hline High & 5 & 8.93 & 20 & 35.09 & 16 & 29.63 & & & \\
\hline
\end{tabular}


Table 3. Cont.

\begin{tabular}{|c|c|c|c|c|c|c|c|c|c|}
\hline & \multicolumn{2}{|c|}{$\begin{array}{l}\text { Mental Health } \\
\text { Professionals } \\
\quad(n=56)\end{array}$} & \multicolumn{2}{|c|}{$\begin{array}{l}\text { Physicians } \\
\text { Working Closely } \\
\text { with COVID-19 } \\
\text { Patients }(n=57)\end{array}$} & \multicolumn{2}{|c|}{$\begin{array}{c}\text { Physicians not } \\
\text { Working Closely } \\
\text { with COVID-19 } \\
\text { Patients }(n=54)\end{array}$} & \multirow[b]{2}{*}{$x^{2}$} & \multirow[b]{2}{*}{$d f$} & \multirow[b]{2}{*}{$p$} \\
\hline & $n$ & $\%$ & $n$ & $\%$ & $n$ & $\%$ & & & \\
\hline MBI Personal Accomplishment & & & & & & & 22.56 & 4 & $<0.001$ \\
\hline Low & 8 & 14.29 & 29 & 50.88 & 19 & 35.19 & & & \\
\hline Medium & 14 & 25.00 & 16 & 28.07 & 14 & 25.93 & & & \\
\hline High & 34 & 60.71 & 12 & 21.05 & 21 & 38.89 & & & \\
\hline
\end{tabular}

No interactions were found between professional groups and gender, but there was a statistically significant association between professional groups and age range, $\chi^{2}(4)=43.52$, $p<0.001$. This association was moderately strong [47], Cramer's $V=0.361$. In particular, post-hoc analysis using adjusted residuals demonstrated that MHPs were significantly less likely to be in the $\leq 40$ years group $(z=-3.05 ; p=0.021)$ and more likely to be in the $41-50$ years group $(z=3.12 ; p=0.016)$, while HPs not working closely with COVID-19 patients were more likely to be in the $\geq 50$ years group $(z=4.36 ; p<0.001)$. Moreover, HPs working closely with COVID-19 patients were significantly more likely to be in the $\leq 40$ years group $(z=5.52 ; p<0.001)$ and less likely to be in the $\geq 50$ years group $(z=-4.04$; $p=0.001)$.

\subsection{Family Conditions}

As Table 3 shows, most MHPs reported having children $(37,66.07 \%)$. The majority were not living alone before the pandemic $(49,87.50 \%)$ and reported no family separation $(40,81.63 \%)$ as a result of COVID-19. No statistically significant association between professional groups and family separation due to COVID-19 were found, $\chi^{2}(2)=5.88$, $p=0.053$.

\subsection{Work Conditions}

MHPs reported work changes due to COVID-19 in 50\% of cases $(n=28)$, and a worsened relationship with patients due to COVID-19 in $62.50 \%$ of cases $(n=35)$.

We detected a statistically significant association between professional groups and changes in the relationship with patients due to COVID-19, $\chi^{2}(4)=11.11, p=0.025$. The effect size was small, Cramer's $V=0.182$. Post-hoc analysis demonstrated that physicians not working closely with COVID-19 patients were significantly less likely to report worsened relationships with their patients $(z=-2.81 ; p=0.045)$.

\subsection{Psychological Outcomes}

As Table 3 shows, most MHPs reported trait anxiety $(37,66.07 \%)$ but no state anxiety $(38,67.86 \%)$. The majority of MHPs reported no post-traumatic distress $(32,57.14 \%)$, low emotional exhaustion $(29,51.79 \%)$, low depersonalization $(40,71.43 \%)$, and high personal accomplishment (34, 60.71\%).

As Table 4 reports, we found statistically significantly differences in hyperarousal median scores between the different professional groups, $\chi^{2}(2)=19.18, p<0.001$. Subsequent pairwise comparisons revealed that hyperarousal scores were statistically significantly lower in MHPs compared to HPs working closely with COVID-19 patients (statistic $=-39.45, p<0.001$ ) and statistically significantly higher in HPs working closely with COVID-19 patients compared to HPs not working closely with COVID-19 patients (statistic $=23.90, p=0.027$ ). Moreover, we found significant differences in intrusion median scores between the different professional groups, $\chi^{2}(2)=10.18, p=0.006$ (Table 4). Subsequent pairwise comparisons revealed that intrusion scores were statistically sig- 
nificantly lower in MHPs compared to HPs working closely with COVID-19 patients (statistic $=-22.76, p=0.036$ ) and to HPs not working closely with COVID-19 patients (statistic $=-27.14, p=0.009)$. We also found statistically significant differences in total posttraumatic median scores between the different professional groups, $\chi^{2}(2)=8.73, p=0.013$ (Table 4). Subsequent pairwise comparisons revealed that total post-traumatic distress scores were lower in MHPs compared to HPs working closely with COVID-19 patients (statistic $=-26.53, p=0.011$ ).

Table 4. Differences between continuous variables.

\begin{tabular}{|c|c|c|c|c|c|c|c|c|c|}
\hline & \multicolumn{2}{|c|}{$\begin{array}{l}\text { Mental Health } \\
\text { Professionals } \\
\quad(n=56)\end{array}$} & \multicolumn{2}{|c|}{$\begin{array}{c}\text { Physicians Working } \\
\text { Closely with COVID-19 } \\
\text { Patients }(n=57)\end{array}$} & \multicolumn{2}{|c|}{$\begin{array}{c}\text { Physicians not Working } \\
\text { Closely with COVID-19 } \\
\text { Patients }(n=54)\end{array}$} & & & \\
\hline & & & & & & & \multicolumn{3}{|c|}{ Kruskal-Wallis Test } \\
\hline & Med & IQR & Med & IQR & Med & IQR & $x^{2}$ & df & $p$ \\
\hline \multicolumn{10}{|l|}{ STAI-Y } \\
\hline Trait Anxiety & 43.00 & 13.75 & 50.00 & 19.00 & 47.00 & 20.25 & 4.26 & 2 & 0.119 \\
\hline State Anxiety & 34.00 & 12.75 & 40.00 & 13.50 & 39.50 & 13.25 & 5.68 & 2 & 0.058 \\
\hline \multicolumn{10}{|l|}{ IES-R } \\
\hline Hyperarousal & 0.86 & 0.96 & 1.43 & 1.28 & 1.00 & 0.72 & 19.18 & 2 & $<0.001$ \\
\hline Avoidance & 0.50 & 0.75 & 0.75 & 0.88 & 0.75 & 1.50 & 2.55 & 2 & 0.280 \\
\hline Intrusion & 1.00 & 0.94 & 1.75 & 1.25 & 1.50 & 1.50 & 10.18 & 2 & 0.006 \\
\hline TOT & 18.00 & 17.50 & 29.00 & 23.00 & 22.50 & 21.00 & 8.73 & 2 & 0.013 \\
\hline \multicolumn{10}{|l|}{ MBI } \\
\hline $\begin{array}{l}\text { Emotional } \\
\text { exhaustion }\end{array}$ & 13.00 & 17.00 & 20.00 & 24.00 & 19.00 & 23.00 & 4.44 & 2 & 0.109 \\
\hline Depersonalization & 2.00 & 4.00 & 7.00 & 7.00 & 3.50 & 8.00 & 18.14 & 2 & $<0.001$ \\
\hline $\begin{array}{c}\text { Personal } \\
\text { accomplishment }\end{array}$ & 39.00 & 10.00 & 29.00 & 14.00 & 34.50 & 44.00 & 21.43 & 2 & $<0.001$ \\
\hline
\end{tabular}

For the MBI, we found statistically significant differences in depersonalization median scores between the different professional groups, $\chi^{2}(2)=8.25, p<0.001$ (Table 4). Subsequent pairwise comparisons revealed that depersonalization scores were lower in MHPs compared to HPs working closely with COVID-19 patients (statistic $=-38.27, p<0.001$ ) and HPs not working closely with COVID-19 patients (statistic $=-23.22, p=0.034$ ). There was also a statistically significant association between professional groups and depersonalization levels, $\chi^{2}(4)=23.54, p<0.001$ (Table 3). This association was moderately strong, Cramer's $V=0.265$. Post-hoc analysis demonstrated that MHPs were significantly more likely to report low depersonalization $(z=3.99 ; p=0.001)$ and less likely to report high depersonalization $(z=-3.33 ; p=0.008)$. Moreover, physicians working closely with COVID-19 patients were less likely to report low depersonalization $(z=-4.02 ; p=0.001)$.

We found statistically significant differences in personal accomplishment between the different professional groups, $\chi^{2}(2)=21.43, p<0.001$ (Table 4). Subsequent pairwise comparisons revealed that personal accomplishment scores were higher in MHPs compared to HPs working closely with COVID-19 patients (statistic $=42.08, p<0.001$ ). Moreover, we detected a statistically significant association between professional groups and personal accomplishment levels, $\chi^{2}(4)=22.56, p<0.001$ (Table 3). The association was moderately strong, Cramer's $V=0.260$. Post-hoc analysis demonstrated that MHPs were more likely to report high personal accomplishment $(z=3.86 ; p=0.001)$ and less likely to report low personal accomplishment $(z=-3.74 ; p=0.002)$. Conversely, physicians working closely with COVID-19 patients were significantly less likely to report high personal accomplishment 
$(z=-3.62 ; p=0.003)$ and more likely to report low personal accomplishment $(z=3.42$; $p=0.006)$.

\subsection{Factors Associated with Anxiety, Post-Traumatic Stress, and Burnout}

Tables 5 and 6 report the results of the multivariable logistic regressions performed to ascertain the effects of possible risk factors on the likelihood of exhibiting psychological outcomes.

Table 5. Multivariable logistic regressions on state anxiety and post-traumatic stress.

\begin{tabular}{|c|c|c|c|c|}
\hline & \multicolumn{2}{|c|}{ STAI-Y State Anxiety } & \multicolumn{2}{|c|}{ IES-R TOT } \\
\hline & OR $(95 \%$ CI) & $p$ & OR $(95 \% \mathrm{CI})$ & $p$ \\
\hline \multicolumn{5}{|l|}{ Socio-Demographic Characteristics } \\
\hline \multicolumn{5}{|l|}{ Group } \\
\hline Physicians not working closely with COVID-19 patients & Ref & & Ref & \\
\hline Physicians working closely with COVID-19 patients & $0.46(0.13-1.66)$ & 0.238 & $0.96(0.30-3.05)$ & 0.984 \\
\hline Mental health professionals & $0.14(0.04-0.49)$ & 0.002 & $0.43(0.14-1.30)$ & 0.134 \\
\hline \multicolumn{5}{|l|}{ Age range } \\
\hline$\leq 40$ years & Ref & & Ref & \\
\hline $41-50$ years & $2.47(0.67-9.10)$ & 0.174 & $3.07(0.89-10.54)$ & 0.075 \\
\hline$\geq 50$ years & $0.67(0.18-2.55)$ & 0.558 & $1.24(0.35-4.41)$ & 0.741 \\
\hline \multicolumn{5}{|l|}{ Gender } \\
\hline Female & Ref & & Ref & \\
\hline Male & $0.17(0.06-0.52)$ & 0.002 & $0.51(0.19-1.38)$ & 0.185 \\
\hline \multicolumn{5}{|l|}{ Family Conditions } \\
\hline \multicolumn{5}{|l|}{ Children } \\
\hline No & Ref & & Ref & \\
\hline Yes & $0.29(0.09-0.88)$ & 0.029 & $1.58(0.57-4.43)$ & 0.381 \\
\hline \multicolumn{5}{|l|}{ Family separation due to COVID-19 } \\
\hline No & Ref & & Ref & \\
\hline Yes & $1.16(0.40-3.40)$ & 0.785 & $4.70(1.73-12.82)$ & 0.002 \\
\hline \multicolumn{5}{|l|}{ Working Conditions } \\
\hline \multicolumn{5}{|l|}{ Work changes due to COVID-19 } \\
\hline Not at all & Ref & & Ref & \\
\hline Slightly & $0.49(0.14-1.75)$ & 0.275 & $0.79(0.24-2.62)$ & 0.695 \\
\hline Considerably & $1.11(0.35-3.55)$ & 0.860 & $1.02(0.34-3.07)$ & 0.972 \\
\hline Extremely & $0.50(0.09-2.92)$ & 0.442 & $1.18(0.22-6.24)$ & 0.849 \\
\hline \multicolumn{5}{|l|}{ Changes in the relationship with patients due to COVID-19 } \\
\hline Not at all & Ref & & Ref & \\
\hline Improved & $3.27(0.76-13.96)$ & 0.110 & $0.70(0.19-2.63)$ & 0.600 \\
\hline Worsened & $1.32(0.37-4.71)$ & 0.673 & $0.73(0.23-2.33)$ & 0.597 \\
\hline \multicolumn{5}{|l|}{ Psychological Characteristics } \\
\hline \multicolumn{5}{|l|}{ STAI-Y Trait Anxiety } \\
\hline No & Ref & & Ref & \\
\hline Yes & $23.70(6.46-86.93)$ & $<0.001$ & $11.84(3.07-45.62)$ & $<0.001$ \\
\hline
\end{tabular}


Table 6. Multivariable logistic regressions on burnout.

\begin{tabular}{|c|c|c|c|c|c|c|}
\hline & \multicolumn{2}{|c|}{ MBI Emotional Exhaustion } & \multicolumn{2}{|c|}{ MBI Depersonalization } & \multicolumn{2}{|c|}{$\begin{array}{c}\text { MBI Personal } \\
\text { Accomplishment }\end{array}$} \\
\hline & OR $(95 \% \mathrm{CI})$ & $p$ & OR $(95 \% \mathrm{CI})$ & $p$ & OR $(95 \% \mathrm{CI})$ & $p$ \\
\hline \multicolumn{7}{|l|}{$\begin{array}{l}\text { Socio-Demographic } \\
\text { characteristics }\end{array}$} \\
\hline \multicolumn{7}{|l|}{ Group } \\
\hline $\begin{array}{l}\text { Physicians not working closely } \\
\text { with COVID-19 patients }\end{array}$ & Ref & & Ref & & Ref & \\
\hline $\begin{array}{l}\text { Physicians working closely with } \\
\text { COVID-19 patients }\end{array}$ & $0.43(0.14-1.31)$ & 0.138 & $0.53(0.16-1.80)$ & 0.312 & $1.49(0.53-4.18)$ & 0.452 \\
\hline Mental health professionals & $0.39(0.14-1.12)$ & 0.080 & $0.33(0.09-1.20)$ & 0.093 & $0.30(0.09-0.95)$ & 0.041 \\
\hline \multicolumn{7}{|l|}{ Age range } \\
\hline$\leq 40$ years & Ref & & Ref & & Ref & \\
\hline $41-50$ years & $2.17(0.68-6.89)$ & 0.188 & $0.33(0.09-1.19)$ & 0.090 & $0.39(0.12-1.12)$ & 0.108 \\
\hline$\geq 50$ years & $1.28(0.40-4.15)$ & 0.678 & $0.35(0.10-1.24)$ & 0.104 & $0.69(0.23-2.13)$ & 0.521 \\
\hline \multicolumn{7}{|l|}{ Gender } \\
\hline Female & Ref & & Ref & & Ref & \\
\hline Male & $0.90(0.36-2.25)$ & 0.825 & $1.78(0.65-4.90)$ & 0.265 & $1.10(0.45-2.71)$ & 0.828 \\
\hline \multicolumn{7}{|l|}{ Family Conditions } \\
\hline \multicolumn{7}{|l|}{ Children } \\
\hline No & Ref & & Ref & & Ref & \\
\hline Yes & $0.31(0.12-0.78)$ & 0.013 & $0.41(0.15-1.12)$ & 0.080 & $0.62(0.24-1.58)$ & 0.318 \\
\hline \multicolumn{7}{|l|}{$\begin{array}{l}\text { Family separation due to } \\
\text { COVID-19 }\end{array}$} \\
\hline No & Ref & & Ref & & Ref & \\
\hline Yes & $1.30(0.51-3.36)$ & .584 & $2.89(0.99-8.40)$ & 0.052 & $1.63(0.62-4.27)$ & 0.320 \\
\hline \multicolumn{7}{|l|}{ Working Conditions } \\
\hline \multicolumn{7}{|l|}{ Work changes due to COVID-19 } \\
\hline Not at all & Ref & & Ref & & Ref & \\
\hline Slightly & $2.36(0.71-7.79)$ & 0.159 & $1.61(0.40-6.58)$ & 0.505 & $2.02(0.63-6.42)$ & 0.234 \\
\hline Considerably & $2.40(0.80-7.20)$ & 0.117 & $1.50(0.43-5.28)$ & 0.527 & $0.80(0.26-2.42)$ & 0.690 \\
\hline Extremely & $\begin{array}{c}4.88 \\
(1.04-22.79)\end{array}$ & 0.044 & $0.92(0.14-5.89)$ & 0.930 & $2.18(0.48-9.95)$ & 0.315 \\
\hline \multicolumn{7}{|l|}{$\begin{array}{l}\text { Changes in the relationship with } \\
\text { patients due to COVID-19 }\end{array}$} \\
\hline Not at all & Ref & & Ref & & Ref & \\
\hline Improved & $0.74(0.21-2.70)$ & 0.654 & $0.39(0.09-1.72)$ & 0.214 & $0.87(0.24-3.15)$ & 0.827 \\
\hline Worsened & $1.07(0.34-3.31)$ & 0.912 & $0.57(0.16-2.04)$ & 0.388 & $0.82(0.26-2.54)$ & 0.728 \\
\hline \multicolumn{7}{|l|}{ PSychological Characteristics } \\
\hline \multicolumn{7}{|l|}{ STAI-Y Trait Anxiety } \\
\hline No & Ref & & Ref & & Ref & \\
\hline Yes & $\begin{array}{c}6.27 \\
(2.23-17.58)\end{array}$ & $<0.001$ & $\begin{array}{c}5.62 \\
(1.51-20.89)\end{array}$ & 0.010 & $2.86(1.10-7.44)$ & 0.031 \\
\hline
\end{tabular}


The logistic regression model for state anxiety was statistically significant, $\chi^{2}(13)=65.07$, $p<0.001$. The model explained $48.86 \%$ (Nagelkerke $R^{2}$ ) of the variance in state anxiety and correctly classified $75.69 \%$ of cases. Its sensitivity was $72.13 \%$, specificity $78.31 \%$, positive predictive value $70.97 \%$, and negative predictive value $79.27 \%$. In particular, MHPs had 0.14 times lower odds of exhibiting state anxiety compared to HPs not working closely with COVID-19 patients ( $p=0.002)$; males had 0.17 times lower odds of exhibiting state anxiety compared to females $(p=0.002)$; and professionals with children had 0.29 times lower odds of exhibiting state anxiety compared to professionals without children $(p=0.029)$. Finally, professionals who reported trait anxiety had 23.70 times higher odds of exhibiting state anxiety compared to those who reported no trait anxiety $(p<0.001)$.

The logistic regression model for post-traumatic stress was statistically significant, $\chi^{2}(13)=39.65, p<0.001$. The model explained 33.84\% (Nagelkerke $R^{2}$ ) of the variance in post-traumatic symptoms and correctly classified $77.08 \%$ of cases. Its sensitivity was $51.11 \%$, specificity $88.89 \%$, positive predictive value $67.65 \%$, and negative predictive value $80.00 \%$. In particular, workers whose family separated because of COVID-19 had 4.70 times higher odds of exhibiting post-traumatic symptoms compared to workers whose families did not separate $(p=0.002)$; and professionals who reported trait anxiety had 11.84 times higher odds of exhibiting post-traumatic symptoms compared to those who reported no trait anxiety $(p=0.001)$.

The logistic regression model for emotional exhaustion was statistically significant, $\chi^{2}(13)=29.78, p=0.005$. The model explained $25.77 \%$ (Nagelkerke $R^{2}$ ) of the variance in emotional exhaustion and correctly classified $72.92 \%$ of cases. Its sensitivity was $50.00 \%$, specificity $85.11 \%$, positive predictive value $64.10 \%$, and negative predictive value $76.19 \%$. In particular, professionals with children had 0.31 times lower odds of exhibiting emotional exhaustion compared to professionals without children $(p=0.013)$; and workers who reported extreme changes in their work due to COVID-19 had 4.88 times higher odds of exhibiting emotional exhaustion compared to workers who reported no changes $(p=0.044)$. Finally, professionals who reported trait anxiety had 6.27 times higher odds of exhibiting emotional exhaustion compared to those who reported no trait anxiety $(p<0.001)$.

The logistic regression model for depersonalization was statistically significant, $\chi^{2}(13)=30.63, p=0.004$. The model explained $29.33 \%$ (Nagelkerke $R^{2}$ ) of the variance in depersonalization and correctly classified $77.78 \%$ of cases. Its sensitivity was $21.88 \%$, specificity $93.75 \%$, positive predictive value $50.00 \%$, and negative predictive value $80.77 \%$. In particular, participants who lived separated from their families during the pandemic had 2.89 times higher odds to exhibit depersonalization compared to those whose families did not separate $(p=0.052)$ and professionals who reported trait anxiety had 5.62 times higher odds to exhibit depersonalization compared to those who reported no trait anxiety $(p=0.010)$.

The logistic regression model for personal accomplishment was statistically significant, $\chi^{2}(13)=30.35, p=0.004$. The model explained $26.60 \%$ (Nagelkerke $R^{2}$ ) of the variance in personal accomplishment and correctly classified $70.81 \%$ of cases. Its sensitivity was $41.30 \%$, specificity $84.69 \%$, positive predictive value $55.88 \%$, and negative predictive value $75.45 \%$. In particular, MHPs had 0.30 times lower odds of exhibiting low personal accomplishment compared to HPs not working closely with COVID-19 patients $(p=0.041)$; moreover, professionals who reported trait anxiety had 2.86 times higher odds of exhibiting low personal accomplishment compared to those who reported no trait anxiety $(p=0.031)$.

\section{Discussion}

This study investigated anxiety, post-traumatic distress, and burnout in a sample of MHPs, comparing their distress to that experienced by other HPs, and focusing on potential sociodemographic, family, and occupational factors.

Most MHPs were female and aged $\geq 41$ years. They mostly reported having children, not living alone before the pandemic, and not experiencing family separation as a result of COVID-19. Consistent with the literature [48,49], they also reported considerable or 
extreme work changes due to COVID-19 in 50\% of cases, and a worsened relationship with patients due to COVID-19 in $62.50 \%$ of cases. Indeed, COVID-19 patients need both comprehensive and specific medical treatment, so the outbreak brings great challenges to HCWs. This may have worsened the relationship between HCWs and their patients. When the survey was administered, care for COVID-19 patients was primarily medical, without the integration of psychological care. It is, therefore, unsurprising that HCWs who did not work closely with COVID-19 patients were significantly less likely to report worsened relationships with patients compared to professionals working closely with COVID-19 patients. Indeed, previous research has pointed out that during the COVID-19 emergency, establishing a functional relationship is difficult for both clinicians and COVID-19 patients, yet good communication can help patients share their burden and recognize that they are not suffering alone [50]. However, even if MHPs did not work with COVID-19 patients, they did not show differences in the perceived changes in their relationship with their patients compared to physicians working closely with COVID-19 patients. This could be explained by most MHPs working remotely during the first phase of the pandemic, representing a significant change in their clinical setting. Indeed, some sensory and bodily characteristics of the face-to-face psychological and psychotherapeutic setting cannot be fully replicated in an online setting [51,52]. In particular, we should consider the impact of virtually getting into patients' houses and letting patients into one's own home, the slight delay in feedbacks from facial microexpressions and the sound of the patient's voice during videocalls, the different visual perspective, and the different interpersonal distance from patients.

Regarding psychological outcomes, MHPs reported good overall mental health except for trait anxiety. In this context, previous research has shown that traumatic experiences can induce changes in trait variables [53], particularly in trait anxiety [54,55]. Thus, the high trait anxiety in our sample could be interpreted as an effect of the pandemic itself.

Most MHPs reported no post-traumatic distress, and their post-traumatic symptom scores were statistically significantly lower compared to HPs working closely with COVID-19 patients. Meanwhile, hyperarousal scores were statistically significantly lower in MHPs compared to HPs working closely with COVID-19 patients, whose scores were also statistically significantly higher compared to those not working closely with COVID-19 patients. Moreover, intrusion scores were statistically significantly lower in MHPs compared to all other professionals. Regarding MBI scores, MHPs reported low emotional exhaustion in $51.79 \%$ of cases, low depersonalization in $71.43 \%$ of cases, and high personal accomplishment in $60.71 \%$ of cases. In particular, contrasting with previous research [32], depersonalization scores were statistically significantly lower in MHPs compared to all other HPs, while personal accomplishment scores were statistically significantly higher in MHPs compared to HPs working closely with COVID-19 patients. Moreover, multivariable logistic regressions showed that MHPs had lower odds of exhibiting state anxiety and low personal accomplishment compared to HPs not working closely with COVID-19 patients.

It seems that MHPs can rely on good affective regulation strategies, allowing them to be more emotionally responsive and cognitively present in clinical practice. In other words, it seems that a mind trained to think as if bombs are falling down should be a fundamental affective resource when the bombs are, in fact, falling down. This invites reflection on the importance of MHPs' personal and professional training and on the specific impact on MHPs' mental functioning of work in which steps are not connected to concrete, objective data. Thus, MHPs can be a key resource in health organizations' decision-making processes regarding patients' care [56,57]. However, we must keep in mind that during the first phase of the pandemic, most MHPs did not work with COVID-19 patients: thus, we have to consider and implement policies aimed at preserving MHPs' mental health. Indeed, though not every professional is needed at the forefront of managing a pandemic, it is vital to preserve a field in which different psychological functions may express and evolve.

As expected, professionals who reported trait anxiety had higher odds of exhibiting state anxiety, post-traumatic symptoms, emotional exhaustion, depersonalization, and 
low personal accomplishment compared to professionals who reported no trait anxiety. Previous literature suggests that anxiety can accentuate post-traumatic stress reactions: individuals with trait anxiety may respond more extremely to a traumatic stressor, distressed by not only the event but also their own reactions $[58,59]$.

Family separation was a significant predictor for post-traumatic symptoms and depersonalization, consistently with previous research suggesting that physical separation or infrequent communication were important sources of distress for HCWs and their families $[60,61]$. Gender was a significant predictor only for state anxiety. The role of gender with respect to anxiety has already been widely investigated: several studies have shown that women are at greater risk of developing mental health problems, especially anxiety and depression, during the COVID-19 pandemic $[20,62,63]$. Moreover, contrary to previous research [64,65], professionals with children reported lower odds of exhibiting state anxiety and emotional exhaustion compared to professionals without children. It seems that the potential distress connected to taking care of children, particularly with homeschooling and changes in daily routines, is lower than the emotional benefits of the parent-child bond. It would be interesting to explore if this association is influenced by child age, romantic relationship with the partner, and affects shared within the family.

Finally, workers who reported an improved relationship with patients due to COVID19 showed higher odds of exhibiting state anxiety compared to those who reported no change in this relationship. It is plausible that those professionals able to describe changes in the quality of their affective bond with patients are also more aware of their own emotional responses, including anxiety.

The health emergency we are experiencing due to the spread of COVID-19 has strongly influenced the psychological and physical health of the general population, including that of HPs. As the mental healthcare demand is especially high during these times and MHPs have to shoulder this responsibility, there is potential for their stress to increase, with adverse effects on their own mental health. However, our data suggest that MHPs' mental health has been almost preserved in the first phase of the pandemic, and it would be useful to rely on these professionals going forward, especially for structuring interventions to improve and support the mental health of the general population and of other HCWs.

\section{Limitations and Future Directions}

This study has some critical limitations. First, the generalizability of results is limited by our small sample derived from one local health authority in North Italy. It would be interesting to compare our results to those detected in other healthcare systems or territories with a different pandemic situation. Second, the cross-sectional design does not allow for causal inferences, so we should be cautious of interpreting the findings as evidence of predictive links between the studied variables. Longitudinal studies are needed to explore the development of distress in HPs-particularly in MHPs-over time and its association with other clinical, family, and occupational variables. Finally, psychological variables were assessed through self-report measures, so further studies should also consider clinical and observational data.

\section{Conclusions}

Despite its limitations, this study is the first attempt to reveal the impact of the COVID-19 pandemic on MHPs, focusing on state and trait anxiety, post-traumatic stress, and burnout, and on their connections with sociodemographic, family, and occupational factors. Moreover, our data elucidate differences in the impact experienced by MHPs and other HCWs. The emotional distress faced by HCWs during a pandemic is a key public concern. Our results suggest that MHPs have adequate resources to handle the impact of the pandemic, and can, thus, be useful in not only helping patients and their families facing the disease but also supporting other health professional, reducing their emotional burden and helping them provide better care to patients. 
Author Contributions: A.N. contributed to the study design, data collection, data interpretation, and drafting and critical revision of the manuscript. I.G.F. contributed to the study design, data analysis and interpretation, and drafting and critical revision of the manuscript. M.A., M.G., and M.D.S. collected and interpreted the data and participated in drafting the manuscript. R.C., P.L., and N.L.B. contributed to data interpretation and participated in drafting the manuscript. A.G. contributed to data interpretation, making an important clinical and intellectual contribution. All authors have read and agreed to the published version of the manuscript.

Funding: This research received no external funding.

Institutional Review Board Statement: The study was conducted according to the guidelines of the Declaration of Helsinki, and approved by the Institutional Review Board of the ASL "Città di Torino," Turin, Italy (on 10 October 2020).

Informed Consent Statement: Informed consent was obtained from all subjects involved in the study.

Data Availability Statement: The datasets generated for this study are available on request from the corresponding author.

Acknowledgments: We are grateful to all survey participants. We express our gratitude to colleagues at the SSS Psicologia Aziendale, ASL Città di Torino, and the Neurology Unit of San Giovanni Bosco Hospital, Turin, for their cooperation and contributions.

Conflicts of Interest: The authors declare no conflict of interest.

\section{References}

1. Granieri, A.; Bonafede, M.; Marinaccio, A.; Iavarone, I.; Marsili, D.; Franzoi, I.G. SARS-CoV-2 and Asbestos Exposure: Can Our Experience with Mesothelioma Patients Help Us Understand the Psychological Consequences of COVID-19 and Develop Interventions? Front. Psychol. 2020, 11, 584320. [CrossRef]

2. Banerjee, D.; Rai, M. Social isolation in Covid-19: The impact of loneliness. Int. J. Soc. Psychiatry 2020, 66, 525-527. [CrossRef]

3. Douglas, P.K.; Douglas, D.B.; Harrigan, D.C.; Douglas, K.M. Preparing for pandemic influenza and its aftermath: Mental health issues considered. Int. J. Emerg. Ment. Health 2009, 11, 137-144.

4. Yang, Y.; Li, W.; Zhang, Q.; Zhang, L.; Cheung, T.; Xiang, Y.-T. Mental health services for older adults in China during the COVID-19 outbreak. Lancet Psychiatry 2020, 7, e19. [CrossRef]

5. Brooks, S.K.; Webster, R.K.; Smith, L.E.; Woodland, L.; Wessely, S.; Greenberg, N.; Rubin, G.J. The psychological impact of quarantine and how to reduce it: Rapid review of the evidence. Lancet 2020, 395, 912-920. [CrossRef]

6. Duan, L.; Zhu, G. Psychological interventions for people affected by the COVID-19 epidemic. Lancet Psychiatry 2020, 7, 300-302. [CrossRef]

7. Colizzi, M.; Bortoletto, R.; Silvestri, M.; Mondini, F.; Puttini, E.; Cainelli, C.; Gaudino, R.; Ruggeri, M.; Zoccante, L. Medically unexplained symptoms in the times of COVID-19 pandemic: A case-report. BBI Health 2020, 5, 100073. [CrossRef] [PubMed]

8. Eisma, M.C.; Lenferink, L.I.M.; Chow, A.Y.M.; Chan, C.L.W.; Li, J. Complicated grief and post-traumatic stress symptom profiles in bereaved earthquake survivors: A latent class analysis. Eur. J. Psychotraumatol. 2019, 10, 1558707. [CrossRef] [PubMed]

9. Eisma, M.C.; Boelen, P.A.; Lenferink, L.I.M. Prolonged grief disorder following the Coronavirus (COVID-19) pandemic. Psychiatry Res. 2020, 288, 113031. [CrossRef] [PubMed]

10. Fekih-Romdhane, F.; Ghrissi, F.; Abbassi, B.; Cherif, W.; Cheour, M. Prevalence and predictors of PTSD during the COVID-19 pandemic: Findings from a Tunisian community sample. Psychiatry Res. 2020, 290, 113131. [CrossRef] [PubMed]

11. Tang, W.; Hu, T.; Hu, B.; Jin, C.; Wang, G.; Xie, C.; Chen, S.; Xu, J. Prevalence and correlates of PTSD and depressive symptoms one month after the outbreak of the COVID-19 epidemic in a sample of home-quarantined Chinese university students. J. Affect. Disord. 2020, 274, 1-7. [CrossRef]

12. Bao, Y.; Sun, Y.; Meng, S.; Shi, J.; Lu, L. 2019-nCoV epidemic: Address mental health care to empower society. Lancet 2020, 395, e-37-e-38. [CrossRef]

13. Garety, P.A.; Kuipers, E.; Fowler, D.; Freeman, D.; Bebbington, P.E. A cognitive model of the positive symptoms of psychosis. Psychol. Med. 2001, 31, 189-195. [CrossRef] [PubMed]

14. Hawryluck, L.; Gold, W.L.; Robinson, S.; Pogorski, S.; Galea, S.; Styra, R. SARS control and psychological effects of quarantine, Toronto, Canada. Emerg. Infect. Dis. 2004, 10, 1206-1212. [CrossRef] [PubMed]

15. Reynolds, D.L.; Garay, J.R.; Deamond, S.L.; Moran, M.K.; Gold, W.; Styra, R. Understanding, compliance and psychological impact of the SARS quarantine experience. Epidemiol. Infect. 2007, 136, 997-1007. [CrossRef] [PubMed]

16. Wang, J.; Lloyd-Evans, B.; Giacco, D.; Forsyth, R.; Nebo, C.; Mann, F.; Johnson, S. Social isolation in mental health: A conceptual and methodological review. Soc. Psych. Psych. Epidemiol. 2017, 52, 1451-1461. [CrossRef] [PubMed]

17. Ahmed, M.A.; Jouhar, R.; Ahmed, N.; Adnan, S.; Aftab, M.; Zafar, M.S.; Khurshid, Z. Fear and practice modifications among dentists to combat novel Coronavirus Disease (COVID-19) outbreak. Int. J. Environ. Res. Public Health 2020, 17, 2821. [CrossRef] 
18. Cao, W.; Fang, Z.; Hou, G.; Han, M.; Xu, X.; Dong, J.; Zheng, J. The psychological impact of the COVID-19 epidemic on college students in China. Psychiatry Res. 2020, 287, 112934. [CrossRef]

19. Desclaux, A.; Badji, D.; Ndione, A.G.; Sow, K. Accepted monitoring or endured quarantine? Ebola contacts' perceptions in Senegal. Soc. Sci. Med. 2017, 178, 38-45. [CrossRef] [PubMed]

20. Moghanibashi-Mansourieh, A. Assessing the anxiety level of Iranian general population during COVID-19 outbreak. Asian J. Psychiatry 2020, 51, 102076. [CrossRef]

21. Li, Z.; Ge, J.; Yang, M.; Feng, J.; Qiao, M.; Jiang, R.; Bi, J.; Zhan, G.; Xu, X.; Wang, L.; et al. Vicarious traumatization in the general public, members, and non-members of medical teams aiding in COVID-19 control. Brain Behav. Immun. 2020, 88, 916-919. [CrossRef]

22. Lima, C.K.T.; Carvalho, P.M.M.; Lima, I.A.A.S.; Nunes, J.V.A.O.; Saraiva, J.S.; de Souza, R.I.; da Silva, C.G.L.; Neto, M.L.R. The emotional impact of Coronavirus 2019-nCoV (new Coronavirus disease). Psychiatry Res. 2020, 287, 112915. [CrossRef] [PubMed]

23. Lee, S.M.; Kang, W.S.; Cho, A.-R.; Kim, T.; Park, J.K. Psychological impact of the 2015 MERS outbreak on hospital workers and quarantined hemodialysis patients. Compr. Psychiatry 2018, 87, 123-127. [CrossRef]

24. Chong, M.-Y.; Wang, W.-C.; Hsieh, W.-C.; Lee, C.-Y.; Chiu, N.-M.; Yeh, W.-C.; Huang, T.-L.; Wen, J.-K.; Chen, C.-L. Psychological impact of severe acute respiratory syndrome on health workers in a tertiary hospital. Br. J. Psychiatry 2004, 185, 127-133. [CrossRef]

25. Dai, Y.; Hu, G.; Xiong, H.; Qiu, H.; Yuan, X. Psychological impact of the coronavirus disease 2019 (COVID-19) outbreak on healthcare workers in China. Lancet Psychiatry 2020, 1. [CrossRef]

26. Lai, J.; Ma, S.; Wang, Y.; Cai, Z.; Hu, J.; Wei, N.; Wu, J.; Du, H.; Chen, T.; Li, R.; et al. Factors associated with mental health outcomes among health care workers exposed to Coronavirus Disease 2019. JAMA Netw. Open 2020, 3, e203976. [CrossRef] [PubMed]

27. Zhang, W.; Wang, K.; Yin, L.; Zhao, W.; Xue, Q.; Peng, M.; Min, B.; Tian, Q.; Leng, H.; Du, J.; et al. Mental health and psychosocial problems of medical health workers during the COVID-19 epidemic in China. Psychother. Psychosom. 2020, 89, 242-250. [CrossRef] [PubMed]

28. Vizheh, M.; Qorbani, M.; Arzaghi, S.M.; Muhidin, S.; Javanmard, Z.; Esmaeili, M. The mental health of healthcare workers in the COVID-19 pandemic: A systematic review. JDMDC 2020, 19, 1967-1978. [CrossRef]

29. Mo, Y.; Deng, L.; Zhang, L.; Lang, Q.; Liao, C.; Wang, N.; Qin, M.; Huang, H. Work stress among Chinese nurses to support Wuhan in fighting against COVID-19 epidemic. J. Nurs. Manag. 2020, 28, 1002-1009. [CrossRef]

30. Naldi, A.; Vallelonga, F.; Di Liberto, A.; Cavallo, R.; Agnesone, M.; Gonella, M.; Sauta, M.D.; Lochner, P.; Tondo, G.; Bragazzi, N.L.; et al. COVID-19 pandemic-related anxiety, distress and burnout: Prevalence and associated factors in healthcare workers of North-West Italy. BJPsych. Open 2021, 7, e27. [CrossRef]

31. Kang, L.; Ma, S.; Chen, M.; Yang, J.; Wang, Y.; Li, R.; Yao, L.; Bai, H.; Cai, Z.; Xiang Yang, B.; et al. Impact on mental health and perceptions of psychological care among medical and nursing staff in Wuhan during the 2019 novel coronavirus disease outbreak: A cross-sectional study. Brain Behav. Immun. 2020, 87, 11-17. [CrossRef] [PubMed]

32. Joshi, G.; Sharma, G. Burnout: A risk factor amongst mental health professionals during COVID-19. Asian. J. Psychiatr. 2020, 54, 102300. [CrossRef] [PubMed]

33. Covid-19-Situazione in Italia. Ministry of Health. Available online: http://www.salute.gov.it/portale/nuovocoronavirus/ dettaglioContenutiNuovoCoronavirus.jsp?lingua=italiano\&id=5351\&area=nuovoCoronavirus\&menu=vuoto (accessed on 1 May 2020).

34. Spielberger, C.D.; Gorsuch, R.L.; Lushene, R.; Vagg, P.R.; Jacobs, G.A. Manual for the State-Trait Anxiety Inventory; Consulting Psychologists Press: Palo Alto, CA, USA, 1983.

35. Pedrabissi, L.; Santinello, M. STAI: State-Trait Anxiety Inventory: Forma Y: Manuale; Organizzazioni Speciali: Florence, Italy, 1996.

36. Pietrantonio, F.; De Gennaro, L.; Di Paolo, M.C.; Solano, L. The impact of event scale. J. Psychosom. Res. 2003, 55, 389-393. [CrossRef]

37. Weiss, D.S.; Marmar, C.R. The Impact of Event Scale-Revised. In Assessing Psychological Trauma and PTSD; Wilson, J.P., Keane, T.M., Eds.; Guilford Press: New York, NY, USA, 1997; pp. 399-411.

38. Maslach, C.; Jackson, S.E. The measurement of experienced burnout. J. Organ. Behav. 1981, 2, 99-113. [CrossRef]

39. Sirigatti, S.; Stefanile, C.; Menoni, E. Per un adattamento italiano del Maslach Burnout Inventory (MBI) [Toward an Italian version of the Maslach Burnout Inventory (MBI)]. Boll. Psicol. Appl. 1988, 187-188, 33-39.

40. El Sawy, A.A. Anxiety level and difficult patients in prosthodontic clinic. J. Am. Sci. 2012, 8, 258-263.

41. Franzoi, I.G.; Sauta, M.D.; Granieri, A. State and trait anxiety among university students: A moderated mediation model of negative affectivity, alexithymia, and housing conditions. Front. Psychol. 2020, 11, 1255. [CrossRef]

42. Creamer, M.; Bell, R.; Failla, S. Psychometric properties of the Impact of Event Scale-Revised. Behav. Res. Ther. 2003, 41, 1489-1496. [CrossRef]

43. Dyrbye, L.N.; West, C.P.; Satele, D.; Boone, S.; Tan, L.; Sloan, J.; Shanafelt, T.D. Burnout among US medical students, residents, and early career physicians relative to the general US population. Acad. Med. 2014, 89, 443-451. [CrossRef]

44. Maslach, C.; Leiter, M.P.; Jackson, S.E. Maslach Burnout Inventory Manual, 4th ed.; Mind Garden Inc.: Menlo Park, CA, USA, 2017.

45. Shanafelt, T.D.; Balch, C.M.; Bechamps, G.; Russell, T.; Dyrbye, L.; Satele, D.; Collicott, P.; Novotny, P.J.; Sloan, J.; Freischlag, J. Burnout and medical errors among American surgeons. Ann. Surg. 2010, 251, 995-1000. [CrossRef] 
46. Dunn, O.J. Multiple comparisons using rank sums. Technometrics 1964, 6, 241-252. [CrossRef]

47. Cohen, J. Statistical Power Analysis for the Behavioral Sciences, 2nd ed.; Psychology Press: New York, NY, USA, 1988.

48. Cao, J.; Wei, J.; Zhu, H.; Duan, Y.; Geng, W.; Hong, X.; Jiang, J.; Zhao, X.; Zhu, B. A study of basic needs and psychological wellbeing of medical workers in the fever clinic of a tertiary general hospital in Beijing during the COVID-19 outbreak. Psychother. Psychosom. 2020, 89, 252-254. [CrossRef]

49. Liu, Q.; Luo, D.; Haase, J.E.; Guo, Q.; Wang, X.Q.; Liu, S.; Xia, L.; Liu, Z.; Yang, J.; Yang, B.X. The experiences of health-care providers during the COVID-19 crisis in China: A qualitative study. Lancet Glob. Health 2020, 8, e790-e798. [CrossRef]

50. Houchens, N.; Tipirneni, R. Compassionate communication amid the COVID-19 pandemic. J. Hosp. Med. 2020, 15, 437-439. [CrossRef] [PubMed]

51. Simpson, S.; Richardson, L.; Pietrabissa, G.; Castelnuovo, G.; Reid, C. Videotherapy and therapeutic alliance in the age of COVID-19. Clin. Psychol. Psychot. 2020, 1-13. [CrossRef]

52. Weinberg, H. Online group psychotherapy: Challenges and possibilities during COVID-19-A practice review. Group Dyn-Theor. Res. 2020, 24, 201. [CrossRef]

53. Hampson, S.E.; Edmonds, G.W.; Goldberg, L.R.; Barckley, M.; Klest, B.; Dubanoski, J.P.; Hillier, T.A. Lifetime trauma, personality traits, and health: A pathway to midlife health status. Psychol. Trauma Theory Res. Pract. Policy 2016, 8, 447-454. [CrossRef] [PubMed]

54. Larsson, M.R.; Bäckström, M.; Johanson, A. The interaction between baseline trait anxiety and trauma exposure as predictor of post-trauma symptoms of anxiety and insomnia. Scand. J. Psychol. 2008, 49, 447-450. [CrossRef]

55. Weems, C.F.; Pina, A.A.; Costa, N.M.; Watts, S.E.; Taylor, L.K.; Cannon, M.F. Predisaster trait anxiety and negative affect predict posttraumatic stress in youths after hurricane Katrina. J. Consult. Clin. Psychol. 2007, 75, 154-159. [CrossRef]

56. Kazak, A.E. Psychology is an essential science: American Psychologist highlights the role of psychology in understanding and addressing COVID-19. Am. Psychol. 2020, 75, 605. [CrossRef]

57. Tullio, V.; Perrone, G.; Bilotta, C.; Lanzarone, A.; Argo, A. Psychological support and psychotherapy via digital devices in Covid-19 emergency time: Some critical issues. Med. Leg. J. 2020, 88, 73-76. [CrossRef]

58. Govêia, C.S.; Cruz, T.; Miranda, D.B.; Guimarães, G.; Ladeira, L.; Tolentino, F.; Amorim, M.; Magalhães, E. Associação entre síndrome de burnout e ansiedade em residentes e anestesiologistas do Distrito Federal [Association between burnout syndrome and anxiety in residents and anesthesiologists of the Federal District]. Rev. Bras. Anestesiol. 2018, 68, 442-446. [CrossRef] [PubMed]

59. Schweizer, T.; Schmitz, J.; Plempe, L.; Sun, D.; Becker-Asano, C.; Leonhart, R.; Tuschen-Caffier, B. The impact of pre-existing anxiety on affective and cognitive processing of a Virtual Reality analogue trauma. PLoS ONE 2017, 12, e0190360. [CrossRef]

60. Montauk, T.R.; Kuhl, E.A. COVID-related family separation and trauma in the intensive care unit. Psychol. Trauma Theory Res. Pract. Policy 2020, 12, S96-S97. [CrossRef]

61. Voo, T.C.; Senguttuvan, M.; Tam, C.C. Family presence for patients and separated relatives during covid-19: Physical, virtual, and surrogate. J. Bioethic. Inq. 2020, 17, 767-772. [CrossRef] [PubMed]

62. Ausín, B.; González-Sanguino, C.; Castellanos, M.Á.; Muñoz, M. Gender-related differences in the psychological impact of confinement as a consequence of COVID-19 in Spain. J. Gender Stud. 2020, 30, 29-38. [CrossRef]

63. Mazza, C.; Ricci, E.; Biondi, S.; Colasanti, M.; Ferracuti, S.; Napoli, C.; Roma, P. A nationwide survey of psychological distress among italian people during the COVID-19 pandemic: Immediate psychological responses and associated factors. Int. J. Environ. Res. Public Health 2020, 17, 3165. [CrossRef] [PubMed]

64. Hacimusalar, Y.; Kahve, A.C.; Yasar, A.B.; Aydin, M.S. Anxiety and hopelessness levels in COVID-19 pandemic: A comparative study of healthcare professionals and other community sample in Turkey. J. Psychiatry Res. 2020, 129, 181-188. [CrossRef]

65. Kuo, F.L.; Yang, P.H.; Hsu, H.T.; Su, C.Y.; Chen, C.H.; Yeh, I.J.; Wu, Y.H.; Chen, L.C. Survey on perceived work stress and its influencing factors among hospital staff during the COVID -19 pandemic in Taiwan. Kaohsiung J. Med. Sci. 2020, 36, 944-952. [CrossRef] 